Childhood-Onset Systemic Lupus Erythematosus. Arthritis Rheumatol. 2018 Apr;70(4):616-624. doi: 10.1002/art.40417. Epub 2018 Mar 2.

Abstract AB1053 Table 1

\begin{tabular}{lccc}
\hline Investigations & Case 1 & Case 2 & Case 3 \\
\hline Haemoglobin (g/L) & 70 & 67 & 70 \\
White cell counts (/L) & $1.5 \times 10^{6}$ & $2.5 \times 10^{6}$ & $3.5 \times 10^{6}$ \\
Lymphocyte count (/L) & $0.4 \times 10^{6}$ & $0.6 \times 10^{6}$ & $0.8 \times 10^{6}$ \\
Platelet count (/L) & $63 \times 10^{9}$ & $50 \times 10^{9}$ & $76 \times 10^{9}$ \\
Urine protein (mg/m $/ \mathrm{hour}$ ) & 74 & & \\
C3 (Normal 50-150 mg/dL) & $<27$ & $<27$ & $<27$ \\
C4 (Normal 20-50 mg/dL) & 4 & 3 & 3 \\
ANA (immunofluorescence method) & $4+$ diffuse & $3+$ diffuse & $4+$ diffuse \\
Anti dsDNA (N: $<60 \mathrm{IU} / \mathrm{mL}$ ) & 707 & 583 & 1394 \\
Serum ferritin (ng/ml) & 3233 & 6767 & 1019 \\
Fasting triglyceride (mg/dl) & 442 & 504 & 328 \\
Fibrinogen (mg/dl) & 120 & 160 & \\
\hline
\end{tabular}

Disclosure of Interests: None declared

DOI: 10.1136/annrheumdis-2019-eular.7017

\section{AB1054 HEPATIC INVOLVEMENT AS A PRESENTATION IN PEDIATRIC LUPUS: A RETROSPECTIVE STUDY OF 3 CASES}

Ankita Singh ${ }^{1}$, Anjani Gummadi ${ }^{1}$, Rakesh Kumar Pilania ${ }^{1}$, Pandiarajan Vignesh ${ }^{1}$, Ankur Jindal ${ }^{1}$, Deepti Suri ${ }^{1}$, Amit Rawat ${ }^{2}$, Surjit Singh ${ }^{1} .{ }^{1}$ Post Graduate Institute of Medical Education and Research, Chandigarh, Advanced Pediatrics Centre, Allergy Immunology Unit, Chandigarh, India; ${ }^{2}$ Post Graduate Institute of Medical Education and Research, Chandigarh, Chandigarh, India

Background: Though abnormal liver tests can be seen during the course of disease in lupus, liver involvement as a presenting manifestation is uncommon in children with lupus (1).

Objectives: We report on 3 children with lupus who had predominant liver involvement at presentation.

Methods: We retrospectively studied 140 pediatric lupus patients from January 1993- November 2018 and collected clinical and laboratory data of patients who had liver involvement as presenting manifestation.

Results: Case 1 was 13-year-old girl with fever and joint pain for 7 months associated with rash and yellow discoloration of eyes and body for 1 month. She also had altered behavior for 3 days. Examination revealed malar rash, pallor, jaundice, bilateral knee arthritis and hepatosplenomegaly. Investigations revealed (table 1) anemia, thrombocytopenia and Iymphopenia. She had conjugated hyperbilirubinemia. Transaminases were elevated. Further investigations confirmed diagnosis of lupus (table 1). Markers for autoimmune hepatitis (SMA; LKM) were negative. She received pulse methylprednisolone followed by tapering doses of oral prednisolone. In view of neurological involvement she also received cyclophosphamide and shifted to azathioprine later. Her initial SLEDAI-2k was 22, that decreased to 4 at 2 year follow-up.

Case 2 was a 11-year-old boy with fever, rash and joint pain for 1 month. Examination revealed pallor, malar rash, oral ulcers, arthritis of bilateral elbow and knee and hepatomegaly. Investigations (table 1) revealed anemia; thrombocytopenia; leucopenia and lymphopenia. In view of multisystem involvement a possibility of lupus was considered which was confirmed by investigations (table 1). He had elevated transaminases. There was no coagulopathy. He was started on oral prednisolone. She also had class 3 lupus nephritis and received pulse cyclophosphamide followed by azathioprine in maintenance. Her transaminases decreased in follow-up. Her initial SLEDAI-2k was 18, that decreased to 2 at 2 year follow-up.

Case 3 was an 9-year-old girl who had rash and abdominal distention for 20 days and fever and altered sensorium for 6 days. On examination she had pallor, malar rash, ascitis and hepatosplenomegaly. Investigations revealed anemia; thrombocytopenia; leucopenia and lymphopenia. She had conjugated hyperbilirubinemia, elevated transaminases and coagulopathy. She also had deranged renal function tests. Ultrasonography reveals coarse echotexture, gross ascitis and splenomegaly. Possibility of lupus with acute on chronic liver decompensation was considered which was confirmed by investigations (Table 1). She was given methyl prednisolone. She had progressive azotemia so possibility of rapidly progressive renal failure was considered and cyclophosphamide was given. However, she developed refractory shock and eventually expired.
Conclusion: Hepatic involvement at presentation in lupus can be multifaceted and poses challenge in diagnosis.

\section{REFERENCES}

[1] Bessone F, Poles N, Roma MG. Challenge of liver disease in systemic lupus erythematosus: Clues for diagnosis and hints for pathogenesis. World J Hepatol 2014 Jun 27; 6(6): 394-409.

Abstract AB1054 Table 1

\begin{tabular}{lccc}
\hline Investigation & Case 1 & Case 2 & Case 3 \\
\hline Haemoglobin (g/L) & 93 & 96 & 75 \\
White cell counts (/L) & $9.5 \times 10^{6}$ & $3.5 \times 10^{6}$ & $4.8 \times 10^{6}$ \\
Lymphocyte count (/L) & $1.9 \times 10^{6}$ & $1.2 \times 10^{6}$ & $0.3 \times 10^{6}$ \\
Platelet count (/L) & $145 \times 10^{9}$ & $147 \times 10^{9}$ & $37 \times 10^{9}$ \\
Total serum bilirubin (mg/dl) & 13.8 & & 7.1 \\
Conjugated bilirubin (mg/dl) & 12 & & 4 \\
Aspartate aminotransferase (IU/L) & 329 & 203 & 69 \\
Alanine aminotransferase (IU/L) & 96 & 156 & 57 \\
Urine protein (mg/m²/hour) & & $24 \mathrm{mg} / \mathrm{m}^{2} / \mathrm{hr}$ & \\
C3 (Normal 50-150 mg/dL) & 68 & 23 & 68 \\
C4 (Normal 20-50 mg/dL) & 4 & 4 & 3 \\
ANA & $3+$ diffuse & $3+$ diffuse & $4+$ speckled \\
Anti dsDNA (N: $<60 \mathrm{IU} / \mathrm{mL}$ ) & 733 & 1621 & 689
\end{tabular}

Disclosure of Interests: None declared

DOI: 10.1136/annrheumdis-2019-eular.6886

\section{AB1055 FINAL DIAGNOSES OF THE PATIENTS WHO WERE REFERRED TO A TERTIARY PEDIATRIC RHEUMATOLOGY OUTPATIENT CLINIC FOR LABORATORY ABNORMALITIES}

Hafize Emine Sonmez, Şerife Gül Karadağ, Ayşe Tanatar, Nuray Aktay Ayaz. University of Health Science, Kanuni Sultan Süleyman Research and Training Hospital, Pediatric Rheumatology, ìstanbul, Turkey

Background: Rheumatology laboratory tests are commonly ordered by clinicians to screen for rheumatic diseases.

Objectives: The study aimed to determine the frequency of use of common rheumatology tests and the final diagnosis of the patients who were referred to a tertiary pediatric rheumatology outpatient clinic for the presence of laboratory abnormalities.

Methods: We prospectively evaluated the patients who were referred due to the abnormal laboratory findings.

Results: A total of 216 patients were examined. Among them, 62 patients had anti-streptomycin $O$ positivity, 47 patients had ANA positivity, 41 patients had elevated acute phase reactants, 16 patients had RF positivity, 9 patients had elevated creatine kinase levels, 2 had lupus anticoagulant positivity. Regarding patients were referred due to carrying MEFV variants $(n=35)$. A diagnosis of rheumatic disease was made in 54 of patients, while the others had non-rheumatic conditions. Final rheumatological disease diagnoses were as follows: familial Mediterranean fever $(n=29)$, juvenile idiopathic arthritis $(n=12)$, systemic lupus erythematosus $(n=4)$, reactive arthritis $(n=3)$, Raynaud's phenomenon $(n=3)$, chronic recurrent multifocal osteomyelitis $(n=1)$, vasculitis $(n=1)$, acute rheumatic fever $(n=1)$.

Conclusion: Diagnosing a rheumatic disease mainly base upon clinical findings rather than laboratory test results. However, clinicians usually prefer to screen rheumatology laboratory tests when patients suffer from non-specific musculoskeletal findings or symptoms such as fatigue. As our study shows most of patients who were referred due to the abnormal laboratory findings, finally diagnosed as having a non-rheumatological condition. Therefore, systemic and careful approach may be needed to reduce unnecessary use of rheumatology laboratory tests.

\section{REFERENCES}

[1] Hashkes PJ, Profile of a pediatric rheumatology practice in Israel, Clin Exp Rheumatol. 2003 Jan-Feb;21(1):123-8.

[2] Rosenberg AM, Analysis of a pediatric rheumatology clinic population. $J$ Rheumatol. 1990 Jun;17(6):827-30.

Acknowledgement: None

Disclosure of Interests: None declared

DOI: 10.1136/annrheumdis-2019-eular.1998 